\title{
Alte Sprachen und Geschichtsunterricht
}

\section{Eine wechselseitige Bereicherung ${ }^{1}$}

Die Ausführungen von Katharina Wesselmann zum Altsprachlichen Unterricht lassen sich grob in zwei große Bereiche aufteilen: 1. in den bezüglich der Sprache und 2. in den bezüglich des Inhalts. Liest man ihre Gedanken im Hinblick auf die Bedeutung für die Geschichtsdidaktik ergeben sich dabei für den ersten Teil der Blick auf die Quellen, ihre Bearbeitung und die notwendige Quellenkritik und etwas nachrangig auch auf die Unterrichtssprache an sich. Für den zweiten Teil kann man die Auswahl der Themen und die Zweckgebundenheit des Faches im gesellschaftlichen Kontext vergleichend betrachten.

\section{Alte Sprachen und historisches Lernen}

„Sed in primis ad fontes ipsos properandum (sc. est) [... “2 - „Aber vor allem muss man zu den Quellen selbst eilen“ so formuliert Erasmus von Rotterdam ein Motto der Humanist*innen und verweist auf die notwendige Rückbesinnung auf das ursprünglich Verfasste. Voraussetzung dafür stellt eine ausreichende Sprachkompetenz (Fremdsprachenund Muttersprachenkenntnis) dar, die die verstehende Originallektüre und Wiedergabe erst ermöglicht. Das Verwenden einer Übersetzung geht immer mit dem Problem einher, dass der*die Lesende nicht genau unterscheiden kann, an welchen Stellen und in welcher Weise der*die Übersetzende eine Interpretation des Originals vorgenommen hat. Diese Manipulation des Ursprungstextes legt oftmals mehr Zeugnis über die Sichtweise des*der Übersetzenden als über die des antiken Autors ab. ${ }^{3}$ Wortwahl, Kürzungen und die Komposition von Textausschnitten erschaffen ein neues Produkt, das selbst zu einer Quelle und einem Dokument seiner Zeit wird, welches mit den Methoden der Quellenkritik interpretiert werden muss. Die Übersetzung, die eigentlich das ursprüng-

1 Kommentierter Beitrag: https://dx.doi.org/10.38072/2703-0784/p4.

2 Erasmus von Rotterdam, De ratione studii ac legendi interpretandique auctores, Paris 1511, in: Desiderii Erasmi Roterodami Opera omnia, hrsg. v. J. H. Waszink u. a., Amsterdam 1971, Vol. I 2, 79-151.

3 Selbst Gefahren für Leib und Leben könnten damit verbunden sein, wie Lebedewa am Beispiel von Dolet zeigt, der sein Leben auf dem Scheiterhaufen verlor, als er mit seiner Übersetzung angeblich Sokrates die Unsterblichkeit der Seele leugnen ließ. Jekatherina Lebedewa, Mit anderen Worten. Die vollkommene Übersetzung bleibt Utopie, https://www.uni-heidelberg.de/presse/ruca/ruca073/wort.html (letzter Zugriff: 26.5.2021). Es wurde hier die männliche Form gewählt, weil es sich im Schulunterricht durchweg um männliche Autoren handelt.

> Das Werk ist unter der Creative-Commons-Lizenz Namensnennung - Weitergabe unter gleichen Bedingungen 4.0 International veröffentlicht. Den Vertragstext finden Sie unter: https://creativecommons.org/licenses/by-sa/4.0/deed.de. Bitte beachten Sie, dass einzelne, entsprechend gekennzeichnete Teile des Werks von der genannten Lizenz ausgenommen sein bzw. anderen urheberrechtlichen Bedingungen unterliegen können. 
lich Verfasste rekonstruieren sollte, muss ihrerseits also wieder dekonstruiert werden. Auch der Blick auf den*die Übersetzende* $n$ in Bezug auf ,gender ${ }^{\star 4}$, ,race ${ }^{{ }^{5}}$ und ,class ${ }^{{ }^{6}}$ und dessen*deren Zeitumstände wären bei exaktem wissenschaftlichem Vorgehen notwendige Untersuchungsgegenstände und müssten kritisch hinterfragt werden. Damit begeben wir uns in einen schwierigen Bereich der Nutzung von Übersetzungen für den Geschichtsunterricht, die als Quellen erkannt oder in die Nähe von Darstellungen geraten würden. Wesselmann plädiert daher in Anlehnung an Erasmus dafür, den Originaltext und damit die Originalsprache zu beherrschen, damit man nur seine eigenen Vorstellungen und die der antiken Autoren kritisch hinterfragen muss, aber nicht auch noch die der übersetzenden Person. Eine solide Sprachkenntnis stellt dazu die notwendige Basis dar. Durchgängige Zweisprachigkeit der abgedruckten Quellen und ihren Übersetzungen wären in diesem Zusammenhang also eine Minimalforderung an ein Geschichtsbuch.

Wesselmann macht auf diese Aspekte mit Blick auf aktuelle politische Strömungen aufmerksam, indem sie bespielhaft auf die ,Red Pillers` verweist, die das Gender-Bild der neuen Rechten in den Vereinigten Staaten von Amerika mit dem Verweis auf antike Texte zu prägen versuchen. ${ }^{7}$ Es sollen damit angebliche Traditionen aufgezeigt werden, die misogynes Verhalten rechtfertigten. Das alleinige Nutzen dieser Texte ergebe ein einseitiges Bild von der Vergangenheit, die dazu noch unkritisch rezipiert werde. Gegen solch monoperspektivisches Vorgehen wendet sich die moderne Geschichtsdidaktik und fordert einen multiperspektivischen Unterricht, der in dem Fall von Wesselmann zumindest verschiedene Übersetzungen, wenn es an der Fremdsprachenkompetenz mangelt, am besten aus verschiedenen Jahrhunderten und Herkunftsländern zu Rate zöge, um auf diese Weise den Schüler*innen einen eigenen kritischen Zugang zu ermöglichen. Die Schulung der Erschließungs ${ }^{8}$ - und Interpretations-

4 Zum,gender-trouble' und der Bedeutung dieser Kategorie allgemein neben, class' und ,race' in der aktuellen Geschichtsdidaktik äußerte sich Lücke kürzlich. Martin Lücke, Gender - Geschichte lernen in einer männlichen Disziplin, in: Sebastian Barsch/Bettina Degner/Christoph Kühberger/Martin Lücke (Hrsg.), Handbuch Diversität im Geschichtsunterricht. Inklusive Geschichtsdidaktik. Frankfurt a. M. 2020, 159-167.

5 ,race' wird nach einem modernen geschichtsdidaktischen Ansatz als „eine natio-ethno-kulturelle Zugehörigkeit betrachtet, die als Kategorie historischen Denkens in Diversität eingebunden werden soll". Lale Yildirim/Martin Lücke, Race als Kategorie historischen Denkens, in: Barsch/Degner/Kühberger/Lücke (wie Anm. 4), 146-158, hier 153.

6 Heuer plädiert für die Rückkehr dieser Kategorie, die aufgrund der marxistischen Konnotation des Begriffs lange Zeit für die Geschichtsdidaktik nicht anschlussfähig erschien. Es könne als Analysekriterium für ein inklusives historisches Lernen nutzbar gemacht werden. Heuer behauptet, dass es eine Fortschreibung bildungsbürgerlicher Traditionen gebe, wodurch es zu einer Exklusion anderer Geschichten aus der unteren Klasse mit geringem kulturellem Kapital (sic!) implizit immer schon gekommen sei. Christian Heuer, Die Kategorie der Klasse im Diskurs der Geschichtsdidaktik, in: Barsch/Degner/Kühberger/Lücke (wie Anm. 4), 135-145. Albert Scherr blickt als Soziologe eher auf die Unterschiede und Ungleichheiten, die er jeweils ausführlich differenziert. Er verweis dabei u. a. auf die Bildungsungleichheit, die in der derzeitigen Wissens- und Informationsgesellschaft von herausragender Bedeutung für die Chancengleichheit sei. Albert Scherr, Kategorie Klasse, in: Barsch/Degner/Kühberger/ Lücke (wie Anm. 4), 117-134, hier 121.

7 Wesselmann in diesem Band: https://dx.doi.org/10.38072/2703-0784/p4.

8 Die Erschließungskompetenz für historische Quellen und Darstellungen bezeichnet kompetentes Handeln im Bereich der Entwicklung, Überprüfung und Darstellung von historischen Sachanalysen anhand von Quellen und Darstellungen und den korrekten und kompetenten Umgang mit verschiedenen Gattungen und führt zu einer eigenständigen Sachanalyse. S. dazu ausführlicher: Peter Gautschi/Jan Hodel/Hans Utz, Kompetenzmodell für «Historisches Lernen» - eine Orientierungshilfe für Lehrerinnen und Lehrer (Fassung August 2009), https://ernst-goebel.hoechst.schule.hessen.de/fach/geschichte/material_ geschichte/allpaed_geschichte/kompetenzorientierunggu/litkompetenzorientierunggu/Gautschi-Kompetenzmodell_fuer_ historisches_LernenAug09.pdf (letzter Zugriff: 26.5.2021). 
kompetenz $z^{9}$ aus dem Kompetenzmodell von Peter Gautschi steht bei einem solchen multivalenten Unterricht im Mittelpunkt. Hier ergeben sich also Synergien der beiden Fächer, die durch weitere Fremdsprachenkenntnisse erweitert werden können.

Da Katharina Wesselmann betont, dass man von bestimmter ideologischer Seite den Alten Sprachen eine distinktive Funktion zuweist, muss dieser Aspekt auch für die Geschichtsdidaktik geprüft werden. In einem heterogenitätssensiblen Geschichtsunterricht sollte Chancengleichheit auch durch den Abbau von Sprachbarrieren ermöglicht werden - eine Exklusion darf nicht stattfinden. Handro unterscheidet hier verschiedene domänenspezifische sprachliche Herausforderungen. Sie konstatiert das Verwischen von Fach- und Alltagssprache, so dass ein sprachliches Vorverständnis die geschichtsspezifische Definition des verwendeten Begriffs erschwert. Daneben können die in den Quellen verwendeten Ausdrücke und Konstruktionen stark vom Sprachgebrauch der Schüler*innen abweichen, so dass durch die Alterität ein Missverstehen die Folge sein kann. ${ }^{10}$ Vor allem Kinder aus nicht-akademischen Haushalten und Familien, in denen Deutsch nicht Erstsprache ist, müssen einen deutlich höheren Aufwand betreiben, um einen solchen Text zu verstehen. ${ }^{11}$ Einen Weg, diesen Herausforderungen entgegenzutreten, bietet $\mathrm{u}$. a. Degner an, indem sie adaptiertes Sprachhandeln favorisiert. ${ }^{12}$ Aber auch im Bereich der Sprachproduktion, die für eine narrative Kompetenz essentiell ist, treten große Unterschiede auf, die ein verstehendes Lesen der von den Schüler*innen verfassten Elaborate erschweren können.

\section{Alte Sprachen und die Inhalte historischen Lernens}

Die Schwerpunktverlagerung des Altsprachlichen Unterricht könne auch in einer veränderten Aufgabenstellung erkannt werden, die nun sprachvergleichend und damit sprachbildend ausgerichtet sei, eine Anpassung an potentielle Adressaten*innen des Faches habe stattgefunden. Da antike Sprachen in der Regel ein freiwählbares Zusatzangebot an Schüler*innen darstellen, ist der Anpassungs- und Legitimationsdruck deutlich spürbarer als für den Geschichtsunterricht. Aber auch hier erfordert die zunehmende Heterogenität eine Umstellung über die dargelegte Sprachsensibilität hinaus. Wenn man nun noch auf den vielfach kulturell eingeschränkten, v.a. eurozentrierten Inhalt des Faches schaut, stellt eine Exklusion der Schüler*innen mit Migrationsgeschichte

9 Unter Interpretationskompetenz wird von Gautschi die Fähigkeit zur Analyse und Deutung, zur Interpretation, zur Herleitung und zum Aufbau sowie zur Darstellung von historischen Sachurteilen im Universum des Historischen bezeichnet. Das Ziel ist die Formulierung eines eigenen Sachurteils. S. dazu ausführlicher: Gautschi/Hodel/Utz, Kompetenzmodell (wie Anm. 8).

10 Vgl. dazu Saskia Handro, Sprache und Diversität im Geschichtsunterricht, in: Barsch/Degner/Kühberger/Lücke (wie Anm. 4), 93-116.

11 Zur Verschiedenartigkeit von Verstehensbarrieren s. Sebastian Barsch/Martin Lücke, Barrierefreiheit bei Quellen und Darstellungen, in: Barsch/Degner/Kühberger/Lücke (wie Anm. 4), 365-374.

12 Bettina Degner, Leichte Sprache und Visualisierung, in: Barsch/Degner/Kühberger/Lücke (wie Anm. 4), 375-384. 
eine durchaus reale Gefahr dar. Da der Geschichtsunterricht aber ein subjektorientiertes Identifikationsangebot allen Adressaten*innen bieten sollte und darüber hinaus durch Erzählen eine Bildung von Gemeinschaft als Ziel verfolgt, ${ }^{13}$ stößt er immer wieder auf Herausforderungen. Es soll, um hier nicht missverstanden zu werden, keine einheitliche historische Identität das Ergebnis sein, die aufgrund von „divided memories“ wohl auch utopisch wäre, ${ }^{14}$ sondern ein gegenseitiges Verstehen auch über „shared memories“, das die Grundlage für eine funktionierende plurale Gesellschaft auf Basis universaler Werte und Normen darstellt. ${ }^{15}$

Man kann also erkennen, dass durchaus ähnliche Herausforderungen im Altsprachlichen Unterricht und im Geschichtsunterricht auftreten, die fächerübergreifend angegangen werden können, wobei das eine Fach für das andere wechselseitig als Hilfswissenschaft zu dienen hat. Interessant wäre ein Blick auf den geschichtskulturellen Bereich ${ }^{16}$, in dem z. B. altsprachliche Inschriften bei Denkmälern, Widmungen oder Bezeichnungen auftauchen, die der betrachtenden Person ohne ausreichende Sprachkenntnis verschlossen blieben. Sie wäre als Rezipient*in auf eine Übersetzung angewiesen, die jedoch, wie dargelegt, die Sichtweise der übersetzenden Person widerspiegelt mit den angedeuteten Folgen für die Konstruktion von Geschichte. Die enge Verknüpfung der beiden Disziplinen kann somit an dem Bereich der Geschichtskultur besonders deutlich gezeigt werden und legt einen verstärkt interdisziplinären Ansatz zum Vorteil beider für die Zukunft nahe.

\section{Autor}

\section{Volker Gaul}

Abgeordnete Lehrkraft für Fachdidaktik der Geschichte an der Christian-Albrechts-Universität zu Kiel. Sein Forschungsschwerpunkt ist die Begabtenförderung im Fach Geschichte.

vgaul@histosem.uni-kiel.de

\footnotetext{
13 Johannes Meyer-Hamme, Historische Identitäten in einer kulturell heterogenen Gesellschaft, in: Michele Barricelli/Martin Lücke (Hrsg.), Handbuch Praxis des Geschichtsunterrichts, Bd. 1. Schwalbach/Ts. 2012, 89-97, hier 94.

14 Vgl. dazu Jan Motte/Rainer Ohliger, Geschichte und Gedächtnis in der Einwanderungsgesellschaft. Migration zwischen historischer Rekonstruktion und Erinnerungspolitik, Essen 2004.

15 Vgl. dazu ausführlich Meyer-Hamme, Historische Identitäten (wie Anm. 13).

16 Als Geschichtskultur wird die „Außenseite" (Schönemann) des Geschichtsbewusstseins bezeichnet, die sich im kollektiven Konstrukt bei Denkmälern, Feiertagen etc. zeigt. Bernd Schönemann, Geschichtsdidaktik, Geschichtskultur, Geschichtswissenschaft. In: Hilke Günther-Arndt/Meik Zülsdorf-Kersting (Hrsg.), Geschichtsdidaktik. Praxishandbuch für die Sekundarstufe I und II, Berlin 2014, 11-23, hier $17 \mathrm{f}$.
} 